IJMMS 28:4 (2001) 223-230

PII. S0161171201006287

http://ijmms.hindawi.com

(c) Hindawi Publishing Corp.

\title{
PROPER CONTRACTIONS AND INVARIANT SUBSPACES
}

\author{
C. S. KUBRUSLY and N. LEVAN
}

(Received 4 December 2000)

\begin{abstract}
Let $T$ be a contraction and $A$ the strong limit of $\left\{T^{* n} T^{n}\right\}_{n \geq 1}$. We prove the following theorem: if a hyponormal contraction $T$ does not have a nontrivial invariant subspace, then $T$ is either a proper contraction of class $\mathscr{C}_{00}$ or a nonstrict proper contraction of class $\mathscr{C}_{10}$ for which $A$ is a completely nonprojective nonstrict proper contraction. Moreover, its self-commutator $\left[T^{*}, T\right]$ is a strict contraction.
\end{abstract}

2000 Mathematics Subject Classification. 47A15, 47B20.

1. Introduction. Let $\mathscr{H}$ be an infinite-dimensional complex Hilbert space. By an operator on $\mathscr{H}$ we mean a bounded linear transformation of $\mathscr{H}$ into itself. The null operator and the identity on $\mathscr{H}$ will be denoted by $O$ and $I$, respectively. If $T$ is an operator, then $T^{*}$ is its adjoint, and $\left\|T^{*}\right\|=\|T\|$. The null space (kernel) of $T$, which is the subspace of $\mathscr{H}$, will be denoted by $\mathcal{N}(T)$. A contraction is an operator $T$ such that $\|T\| \leq 1$ (i.e., $\|T x\| \leq\|x\|$ for every $x$ in $\mathscr{H}$ or, equivalently, $T^{*} T \leq I$ ). A strict contraction is an operator $T$ such that $\|T\|<1$ (i.e., $\sup _{0 \neq x}(\|T x\| /\|x\|)<1$; equivalently, $T^{*} T \prec I$, which means that $T^{*} T \leq \gamma I$ for some $\left.\gamma \in(0,1)\right)$. An isometry is a contraction for which $\|T x\|=\|x\|$ for every $x$ in $\mathscr{H}$ (i.e., $T^{*} T=I$ so that $\|T\|=1$ ).

We summarize below some well-known results on contractions that will be applied throughout (cf. [16, page 40], [5, 9, 10, 11, 13], and [8, Chapter 3]). If $T$ is a contraction, then $T^{* n} T^{n} \stackrel{s}{\rightarrow} A$. That is, the sequence $\left\{T^{* n} T^{n}\right\}_{n \geq 1}$ of operators on $\mathscr{H}$ converges strongly to an operator $A$ on $\mathscr{H}$, which means that $\left\|\left(T^{* n} T^{n}-A\right) x\right\| \rightarrow 0$ for every $x$ in $\mathscr{H}$. Moreover, $A$ is a nonnegative contraction (i.e., $O \leq A \leq I$ ), $\|A\|=1$ whenever $A \neq O, T^{* n} A T^{n}=A$ for every integer $n \geq 1$ (so that $T$ is an isometry if and only if $A=I),\left\|T^{n} x\right\| \rightarrow\left\|A^{1 / 2} x\right\|$ for every $x$ in $\mathscr{H}$, and the null spaces of $A$ and $I-A$, viz. $\mathcal{N}(A)=\{x \in \mathscr{H}: A x=0\}$ and $\mathcal{N}(I-A)=\{x \in \mathscr{H}: A x=x\}$, are given by

$$
\begin{aligned}
\mathcal{N}(A) & =\left\{x \in \mathscr{H}: T^{n} x \longrightarrow 0\right\}, \\
\mathcal{N}(I-A) & =\left\{x \in \mathscr{H}:\left\|T^{n} x\right\|=\|x\| \forall n \geq 1\right\} \\
& =\{x \in \mathscr{H}:\|A x\|=\|x\|\} .
\end{aligned}
$$

Recall that $T$ is a contraction if and only if $T^{*}$ is. Thus $T^{n} T^{* n} \stackrel{s}{\rightarrow} A_{*}$, where $O \leq A_{*} \leq I$, $\left\|A_{*}\right\|=1$ whenever $A_{*} \neq O, T^{n} A_{*} T^{* n}=A_{*}$ for every $n \geq 1$ (so that $T$ is a co-isometryi.e., $T^{*}$ is an isometry-if and only if $A_{*}=I$ ), $\left\|T^{* n} x\right\| \rightarrow\left\|A_{*}^{1 / 2} x\right\|$ for every $x$ in $\mathscr{H}$, and

$$
\begin{aligned}
\mathcal{N}\left(A_{*}\right) & =\left\{x \in \mathscr{H}: T^{* n} x \longrightarrow 0\right\}, \\
\mathcal{N}\left(I-A_{*}\right) & =\left\{x \in \mathscr{H}:\left\|T^{* n} x\right\|=\|x\| \forall n \geq 1\right\} \\
& =\left\{x \in \mathscr{H}:\left\|A_{*} x\right\|=\|x\|\right\} .
\end{aligned}
$$


An operator $T$ on $\mathscr{H}$ is uniformly stable if the power sequence $\left\{T^{n}\right\}_{n \geq 1}$ converges uniformly to the null operator (i.e., $\left\|T^{n}\right\| \rightarrow 0$ ). It is strongly stable if $\left\{T^{n}\right\}_{n \geq 1}$ converges strongly to the null operator (i.e., $\left\|T^{n} x\right\| \rightarrow 0$ for every $x$ in $\mathscr{H}$ ), and weakly stable if $\left\{T^{n}\right\}_{n \geq 1}$ converges weakly to the null operator (i.e., $\left\langle T^{n} x ; y\right\rangle \rightarrow 0$ for every $x, y \in \mathscr{H}$ or, equivalently, $\left\langle T^{n} x ; x\right\rangle \rightarrow 0$ for every $x \in \mathscr{H}$ ). It is clear that uniform stability implies strong stability, which implies weak stability. The converses fail (a unilateral shift is a weakly stable isometry and its adjoint is a strongly stable co-isometry) but hold for compact operators. $T$ is uniformly stable if and only if $T^{*}$ is uniformly stable, and $T$ is weakly stable if and only if $T^{*}$ is weakly stable. However, strong convergence is not preserved under the adjoint operation so that strong stability for $T$ does not imply strong stability for $T^{*}$ (and vice versa). If $T$ is a strongly stable contraction (i.e., if $\mathcal{N}(A)=\mathscr{H}$, which means that $A=O$ ), then it is usual to say that $T$ is a $\mathscr{C}_{0}$.contraction. If $T^{*}$ is a strongly stable contraction (i.e., if $\mathcal{N}\left(A_{*}\right)=\mathscr{H}$, which means that $\left.A_{*}=O\right)$, then $T$ is a $\mathscr{C}_{.0}$-contraction. On the other extreme, if a contraction $T$ is such that $T^{n} x \rightarrow 0$ for every nonzero vector $x$ in $\mathscr{H}$ (i.e., if $\mathcal{N}(A)=\{0\}$ ), then it is said to be a $\mathscr{C}_{1}$.-contraction. Dually, if a contraction $T$ is such that $T^{* n} x \rightarrow 0$ for every nonzero vector $x$ in $\mathscr{H}$ (i.e., if $\mathcal{N}\left(A_{*}\right)=\{0\}$ ), then it is a $\mathscr{C}_{.1}$-contraction. These are the Nagy-Foiaş classes of contractions (see [16, page 72]). All combinations are possible leading to classes $\mathscr{C}_{00}, \mathscr{C}_{01}, \mathscr{C}_{10}$, and $\mathscr{C}_{11}$. In particular, $T$ and $T^{*}$ are both strongly stable contractions if and only if $T$ is of class $\mathscr{C}_{00}$. Generally,

$$
\begin{aligned}
& T \in \mathscr{C}_{00} \Longleftrightarrow A=A_{*}=O, \\
& T \in \mathscr{C}_{01} \Longleftrightarrow A=O, \mathcal{N}\left(A_{*}\right)=\{0\}, \\
& T \in \mathscr{C}_{10} \Longleftrightarrow \mathcal{N}(A)=\{0\}, A_{*}=O, \\
& T \in \mathscr{C}_{11} \Longleftrightarrow \mathcal{N}(A)=\mathcal{N}\left(A_{*}\right)=\{0\} .
\end{aligned}
$$

If $T$ is a strict contraction, then it is uniformly stable, and hence of class $\mathscr{C}_{00}$. Thus, a contraction not in $\mathscr{C}_{00}$ is necessarily nonstrict (i.e., if $T \notin \mathscr{C}_{00}$, then $\|T\|=1$ ). In particular, contractions in $\mathscr{C}_{1}$. or in $\mathscr{C}_{.1}$ are nonstrict.

2. Proper contractions. An operator $T$ is a proper contraction if $\|T x\|<\|x\|$ for every nonzero $x$ in $\mathscr{H}$ or, equivalently, if $T^{*} T<I$. The terms "strict" and "proper" contractions are sometimes interchanged in current literature. We adopt the terminology of [7, page 82] for strict contraction. Obviously, every strict contraction is a proper contraction, every proper contraction is a contraction, and the converses fail: any isometry is a contraction but not a proper contraction, and the diagonal operator $T=\operatorname{diag}\left\{(k+1)(k+2)^{-1}\right\}_{k=0}^{\infty}$ is a proper contraction on $\ell_{+}^{2}$ but not a strict contraction. Thus, proper contractions comprise a class of operators that is properly included in the class of all contractions and properly includes the class of all strict contractions. If $T$ is a proper contraction, then so is $T^{*} T$ (reason: $S T$ is a proper contraction whenever $S$ is a contraction and $T$ is a proper contraction). Thus, the point spectrum $\sigma_{P}\left(T^{*} T\right)$ lies in the open unit disc. If, in addition, $T$ is compact, then so is $T^{*} T$ and hence its spectrum $\sigma\left(T^{*} T\right)$, which is always closed, also lies in the open unit disc (for $\sigma(K) \backslash\{0\}=\sigma_{P}(K) \backslash\{0\}$ whenever $K$ is compact). This implies that the spectral radius $r\left(T^{*} T\right)$ is less than one. Therefore, $\|T\|^{2}=r\left(T^{*} T\right)<1$. 
Conclusion. The concepts of proper and strict contraction coincide for compact operators.

Proper contractions have been investigated in connection with unitary dilations (the minimal unitary dilation of a proper contraction is a bilateral shift whose multiplicity does not exceed the dimension of $\mathscr{H}$ - see [16, page 91]), and also with strong stability of contractive semigroups (cf. [1]). They were further investigated in [15] by considering different topologies in $\mathscr{H}$. Here are three basic properties of proper contractions that will be needed in the sequel.

Proposition 2.1. $T$ is a proper contraction if and only if $T^{*}$ is a proper contraction.

PRoof. Recall that $\left\|T^{*} x\right\|^{2}=\left\langle T^{*} x ; T^{*} x\right\rangle=\left\langle T T^{*} x ; x\right\rangle \leq\left\|T T^{*} x\right\|\|x\|$ for every $x$ in $\mathscr{H}$, for all operators $T$ on $\mathscr{H}$. Take an arbitrary nonzero vector $x$ in $\mathscr{H}$. If $T^{*} x=0$, then $\left\|T^{*} x\right\|<\|x\|$ trivially. On the other hand, if $T^{*} x \neq 0$ and $T$ is a proper contraction, then $\left\|T T^{*} x\right\|<\left\|T^{*} x\right\| \neq 0$ so that $\left\|T^{*} x\right\|^{2}<\left\|T^{*} x\right\|\|x\|$, and hence $\left\|T^{*} x\right\|<\|x\|$. That is, $T^{*}$ is a proper contraction. Dually, since $T^{* *}=T$, it follows that $T$ is a proper contraction whenever $T^{*}$ is.

If $S$ is a contraction and $T$ is a proper contraction, then $S T$ is a proper contraction (as we have already seen above) and so is $S^{*} T^{*}$ by Proposition 2.1. Another application of Proposition 2.1 ensures that $T S=\left(S^{*} T^{*}\right)^{*}$ is still a proper contraction. Summing up: left or right product of a contraction and a proper contraction is again a proper contraction.

PROPOSITION 2.2. Every proper contraction is weakly stable.

Proof. If $\|T x\|<\|x\|$ for every nonzero $x$ in $\mathscr{H}$, then $T$ is completely nonisometric (i.e., there is no nonzero reducing subspace $\mathcal{M}$ for $T$ such that $\left\|T^{n} x\right\|=\|x\|$ for every $x \in \mathcal{M}$ and every $n \geq 1$ ), and therefore completely nonunitary. But a completely nonunitary contraction is weakly stable. In fact, the Foguel decomposition for contractions says that every contraction is the direct sum of a weakly stable contraction and a unitary operator (cf. [6, page 55] or [8, page 106]).

The converse of Proposition 2.2 fails: shifts are weakly stable isometries. However, as it was raised in [1], a proper contraction is not necessarily strongly stable. Indeed, if $T$ is the weighted unilateral shift $T=\operatorname{shift}\left\{(k+1)^{1 / 2}(k+2)^{-1}(k+3)^{1 / 2}\right\}_{k=0}^{\infty}$ on $\ell_{+}^{2}$, which is a proper contraction because $(k+1)(k+2)^{-2}(k+3)<1$ for every $k \geq 0$, then $A$ is the diagonal operator $A=\operatorname{diag}\left\{(k+1)(k+2)^{-1}\right\}_{k=0}^{\infty} \neq O$ (cf. [10] or [8, pages 51, 52]) so that $T$ is not strongly stable. As a matter of fact, $\mathcal{N}(A)=\{0\}$ and (as it is readily verified) $A_{*}=O$. Hence $T$ is a proper contraction of class $\mathscr{C}_{10}$. The converse is much simpler: strongly stable contractions are not necessarily proper contractions. For instance, a backward unilateral shift $S_{+}^{*}$ is a strongly stable co-isometry (in fact, an operator is a strongly stable co-isometry if and only if it is a backward unilateral shift). Thus $S_{+}^{*}$ is a strongly stable contraction but not a proper contraction (it is a nonproper contraction of class $\left.\mathscr{C}_{01}\right)$. Actually, even $a \mathscr{b}_{00}$-contraction is not necessarily a proper contraction. For example, the weighted bilateral shift $T=\operatorname{shift}\left\{(|k|+1)^{-1}\right\}_{k=-\infty}^{\infty}$ on $\ell^{2}$ is a contraction of class $\mathscr{C}_{00}$ (reason: $\prod_{k=0}^{n}(|k|+1)^{-1}=(n !)^{-1} \rightarrow 0$ as $n \rightarrow \infty$, which means that both products $\prod_{k=0}^{\infty}(|k|+1)^{-1}$ and $\prod_{k=-\infty}^{0}(|k|+1)^{-1}$ diverge to 
0-see [3, page 181]) but not a proper contraction because $(|k|+1)^{-1}=1$ for $k=0$. It is worth noticing that the weighted bilateral shift $T=\operatorname{shift}\left\{1-(|k|+2)^{-2}\right\}_{k=-\infty}^{\infty}$ on $\ell^{2}$ is a proper contraction of class $\mathscr{C}_{11}$. Indeed, $0<1-(|k|+2)^{-2}<1$ for each integer $k$, and both products $\prod_{k=0}^{\infty}\left(1-(|k|+2)^{-2}\right)$ and $\prod_{k=-\infty}^{0}\left(1-(|k|+2)^{-2}\right)$ do not diverge to 0 (cf. [3, page 181] again) - these products converge once the series $\sum_{k=0}^{\infty}(|k|+2)^{-2}$ converges.

Proposition 2.3. If $T$ is a proper contraction, then $A$ is a proper contraction.

Proof. Let $T$ be a proper contraction and take an arbitrary nonzero vector $x$ in $\mathscr{H}$. If $T^{m} x=0$ for some $m \geq 1$, then $T^{n} x=0$ for every integer $n \geq m$. If $T^{n} x \neq 0$ for every integer $n \geq 1$, then $\left\|T^{n+1} x\right\|=\left\|T T^{n} x\right\|<\left\|T^{n} x\right\|<\|x\|$ so that $\left\{\left\|T^{n} x\right\|\right\}_{n \geq 1}$ is a strictly decreasing sequence of positive numbers. In the former case $T$ is trivially strongly stable so that $A=O$ is a trivial proper contraction. In the latter case $\left\{\left\|T^{n} x\right\|\right\}_{n \geq 1}$ converges in the real line to $\left\|A^{1 / 2} x\right\|$ so that $\left\|A^{1 / 2} x\right\|<\|x\|$. Thus $\|A x\| \leq$ $\left\|A^{1 / 2} x\right\|<\|x\|$.

A backward unilateral shift shows that the converse of Proposition 2.3 does not hold true as well (i.e., there exist nonproper contractions $T$ for which $A$ is a proper contraction).

3. Invariant subspaces. A subspace $\mathcal{M}$ of $\mathscr{H}$ is a closed linear manifold of $\mathscr{H} . \mathcal{M}$ is nontrivial if $\{0\} \neq \mathcal{M} \neq \mathscr{H}$. If $T$ is an operator on $\mathscr{H}$ and $T(\mathcal{M}) \subseteq \mathcal{M}$, then $\mathcal{M}$ is invariant for $T$ (or $\mathcal{M}$ is $T$-invariant). If $\mathcal{M}$ is a nontrivial invariant subspace for $T$, then its orthogonal complement $\mathcal{M}^{\perp}$ is a nontrivial invariant subspace for $T^{*}$. If $\mathcal{M}$ is invariant for both $T$ and $T^{*}$ (equivalently, if both $\mathcal{M}$ and $\mathcal{M}^{\perp}$ are $T$-invariant), then $\mathcal{M}$ reduces $T$. A classical open question in operator theory is: does a contraction not in $\mathscr{C}_{00}$ have a nontrivial invariant subspace? Although this is still an unsolved problem we know that the following result holds true.

LEMMA 3.1. If a contraction has no nontrivial invariant subspace, then it is either a $\mathscr{C}_{00}, a \mathscr{C}_{01}$, or a $\mathscr{C}_{10}$-contraction.

Proof. See, for instance, [8, page 71].

The class of contractions $T$ for which $A$ is a projection was investigated in [4, 10]. It coincides with the class of all contractions $T$ that commute with $A$; that is, $A=A^{2}$ if and only if $A T=T A$ (cf. [4]). Equivalently, $\mathcal{N}\left(A-A^{2}\right)=\mathscr{H}$ if and only if $\mathcal{N}(A T-T A)=\mathscr{H}$. The next proposition extends this equivalence.

Proposition 3.2. $\mathcal{N}\left(A-A^{2}\right)$ is the largest subspace of $\mathscr{H}$ that is included in $\mathcal{N}(A T-$ $T A)$ and is $T$-invariant.

Proof. See [10] (or [8, page 52]).

We will say that $A$ is completely nonprojective if $A x \neq A^{2} x$ for every nonzero $x$ in $\mathscr{H}$ (i.e., if $\left.\mathcal{N}\left(A-A^{2}\right)=\{0\}\right)$. Since $\mathcal{N}\left(A-A^{2}\right)$ reduces the selfadjoint operator $A$, this means that no nonzero direct summand of $A$ is a projection. If $A$ is completely nonprojective, then $T$ is a $\mathscr{C}_{1}$.-contraction (for $\mathcal{N}(A) \subseteq \mathcal{N}\left(A-A^{2}\right)$ ). 
LEMMA 3.3. If a contraction $T$ has no nontrivial invariant subspace, then either $T$ is strongly stable or A is a completely nonprojective nonstrict proper contraction.

Proof. Suppose that $T$ is a contraction without a nontrivial invariant subspace. Since $\mathcal{N}\left(A-A^{2}\right)$ is an invariant subspace for $T$ (by Proposition 3.2), it follows that either $\mathcal{N}\left(A-A^{2}\right)=\mathscr{H}$ or $\mathcal{N}\left(A-A^{2}\right)=\{0\}$. In the former case $A$ is a projection (i.e., $A=A^{2}$ ). However, as it was shown in [10], if $A$ is a projection then $T$ is the direct sum of a strongly stable contraction $G$, a unilateral shift $S_{+}$, and a unitary operator $U$, where any of the direct summands of the decomposition

$$
T=G \oplus S_{+} \oplus U
$$

may be missing (see also [8, page 83]). But $T$ has no nontrivial invariant subspace so that $T=G$. That is, $T$ is a strongly stable contraction, for $S_{+}$and $U$ clearly have nontrivial invariant subspaces (isometries have nontrivial invariant subspaces). In the latter case $A$ is a completely nonprojective proper contraction. Indeed, $\{x \in \mathscr{H}:\|A x\|=$ $\|x\|\}=\mathcal{N}(I-A) \subseteq \mathcal{N}\left(A-A^{2}\right)=\{0\}$. Finally, the contraction $A$ is not strict (i.e., $\|A\|=1$ ) whenever $T$ is not strongly stable (i.e., whenever $A \neq O$ ).

Another classical open question in operator theory is: does a hyponormal operator have a nontrivial invariant subspace? Recall that an operator $T$ on $\mathscr{H}$ is hyponormal if $T T^{*} \leq T^{*} T$ (equivalently, if $\left\|T^{*} x\right\| \leq\|T x\|$ for every $x$ in $\mathscr{H}$ ), and $T$ is cohyponormal if $T^{*}$ is hyponormal. Here is a consequence of Lemmas 3.1 and 3.3 for hyponormal contractions. It uses the fact that a cohyponormal contraction $T$ is such that $A$ is a projection. This implies that a completely nonunitary cohyponormal contraction is strongly stable (cf. [9, 12, 14]).

THEOREM 3.4. If a hyponormal contraction $T$ has no nontrivial invariant subspace, then it is either a $\mathscr{C}_{00}$-contraction or a $\mathscr{C}_{10}$-contraction for which $A$ is a completely nonprojective nonstrict proper contraction.

Proof. If $T$ has no nontrivial invariant subspace, then $T^{*}$ has no nontrivial invariant subspace. If $T$ is a contraction, then Lemmas 3.1 and 3.3 ensure that either $A=$ $A_{*}=O, A=O$ and $A_{*}$ is a completely nonprojective nonstrict proper contraction, or $A$ is a completely nonprojective nonstrict proper contraction and $A_{*}=O$. However, if $T$ is hyponormal, then $A_{*}$ is a projection [9] so that $A_{*}=O$ (see also [8, page 78]).

Can the conclusion in Theorem 3.4 be sharpened to $T \in \mathscr{C}_{00}$ ? In other words, does a hyponormal contraction not in $\mathscr{C}_{00}$ have a nontrivial invariant subspace? The question has an affirmative answer if we replace " $\mathscr{C}_{00}$-contraction" with "proper contraction." That is, if a hyponormal contraction is not a proper contraction, then it has a nontrivial invariant subspace. This will be proved in Theorem 3.6 below, but first we consider the following auxiliary result. Let $D$ denote the self-commutator of $T$; that is,

$$
D=\left[T^{*}, T\right]=T^{*} T-T T^{*} .
$$

Thus, a hyponormal is precisely an operator $T$ for which $D$ is nonnegative (i.e., $D \geq O$ ).

Proposition 3.5. If $T$ is a hyponormal contraction, then $D$ is a contraction whose power sequence converges strongly. If $P$ is the strong limit of $\left\{D^{n}\right\}_{n \geq 1}$, then $P T=O$. 
Proof. Take an arbitrary $x$ in $\mathscr{H}$ and an arbitrary nonnegative integer $n$. Suppose that $T$ is hyponormal and let $R=D^{1 / 2} \geq O$ be the unique nonnegative square root of $D \geq O$. If, in addition, $T$ is a contraction, then

$$
\begin{aligned}
\left\langle D^{n+1} x ; x\right\rangle & =\left\|R^{n+1} x\right\|^{2}=\left\langle D R^{n} x ; R^{n} x\right\rangle \\
& =\left\|T R^{n} x\right\|^{2}-\left\|T^{*} R^{n} x\right\|^{2} \\
& \leq\left\|R^{n} x\right\|^{2}-\left\|T^{*} R^{n} x\right\|^{2} \leq\left\|R^{n} x\right\|^{2} \\
& =\left\langle D^{n} x ; x\right\rangle .
\end{aligned}
$$

This shows that $R$ (and so $D$ ) is a contraction: set $n=0$ above. It also shows that $\left\{D^{n}\right\}_{n \geq 1}$ is a decreasing sequence of nonnegative contractions. Since a bounded monotone sequence of selfadjoint operators converges strongly,

$$
D^{n} \stackrel{s}{\longrightarrow} P \geq O \text {. }
$$

Indeed, the strong limit $P$ of $\left\{D^{n}\right\}_{n \geq 1}$ is nonnegative, for the set of all nonnegative operators on $\mathscr{H}$ is weakly (thus strongly) closed. As a matter of fact, $P=P^{2}$ (the weak limit of any weakly convergent power sequence is idempotent) and so $P \geq O$ is a projection. Moreover,

$$
\sum_{n=0}^{m}\left\|T^{*} R^{n} x\right\|^{2} \leq \sum_{n=0}^{m}\left(\left\|R^{n} x\right\|^{2}-\left\|R^{n+1} x\right\|^{2}\right)=\|x\|^{2}-\left\|R^{m+1} x\right\|^{2} \leq\|x\|^{2}
$$

for all $m \geq 0$ so that $\left\|T^{*} R^{n} x\right\| \rightarrow 0$ as $n \rightarrow \infty$. Hence

$$
T^{*} P x=T^{*} \lim _{n} D^{n} x=\lim _{n} T^{*} R^{2 n} x=0
$$

for every $x$ in $\mathscr{H}$, and therefore $P T=O$ (since $P$ is selfadjoint).

THEOREM 3.6. If a hyponormal contraction has no nontrivial invariant subspace, then it is a proper contraction and its self-commutator is a strict contraction.

Proof. (a) Take an arbitrary operator $T$ on $\mathscr{H}$ and an arbitrary $x$ in $\mathscr{H}$. Note that

$$
T^{*} T x=\|T\|^{2} x \text { if and only if }\|T x\|=\|T\|\|x\| .
$$

Indeed, if $T^{*} T x=\|T\|^{2} x$, then $\|T x\|^{2}=\left\langle T^{*} T x ; x\right\rangle=\|T\|^{2}\|x\|^{2}$. Conversely, if $\|T x\|=$ $\|T\|\|x\|$, then $\left\langle T^{*} T x ;\|T\|^{2} x\right\rangle=\|T\|^{4}\|x\|^{2}$, and hence

$$
\begin{aligned}
\left\|T^{*} T x-\right\| T\left\|^{2} x\right\|^{2} & =\left\|T^{*} T x\right\|^{2}-2 \operatorname{Re}\left\langle T^{*} T x ;\|T\|^{2} x\right\rangle+\|T\|^{4}\|x\|^{2} \\
& =\left\|T^{*} T x\right\|^{2}-\|T\|^{4}\|x\|^{2} \leq\left(\left\|T^{*} T\right\|^{2}-\|T\|^{4}\right)\|x\|^{2}=0 .
\end{aligned}
$$

Put $\mathcal{M}=\{x \in \mathscr{H}:\|T x\|=\|T\|\|x\|\}=\mathcal{N}\left(\|T\|^{2} I-T^{*} T\right)$, which is a subspace of $\mathscr{H}$. If $T$ is hyponormal, then $\mathcal{M}$ is $T$-invariant. In fact, if $T$ is hyponormal and $x \in \mathcal{M}$, then

$$
\|T(T x)\| \leq\|T\|\|T x\|=\|\| T\left\|^{2} x\right\|=\left\|T^{*} T x\right\| \leq\|T(T x)\|
$$


and so $T x \in \mathcal{M}$ (see also [6, page 9]). Now let $T$ be a hyponormal contraction. If $\|T\|<1$, then it is trivially a proper contraction. If $\|T\|=1$ and $T$ has no nontrivial invariant subspace, then $\mathcal{M}=\{x \in \mathscr{H}:\|T x\|=\|x\|\}=\{0\}$ (actually, if $\mathcal{M}=\mathscr{H}$, then $T$ is an isometry, and isometries have invariant subspaces). Hence $T$ is a proper contraction.

(b) Let $D \geq O$ be the self-commutator of a hyponormal contraction $T$ and let $P$ be the strong limit of $\left\{D^{n}\right\}_{n \geq 1}$ so that $P T=O$ (cf. Proposition 3.5). Suppose $T$ has no nontrivial invariant subspace. Since $\mathcal{N}(P)$ is a nonzero invariant subspace for $T$ whenever $P T=O$ and $T \neq O$, it follows that $\mathcal{N}(P)=\mathscr{H}$. Hence $P=O$ and so $D$ is strongly stable $\left(D^{n} \stackrel{s}{\rightarrow} O\right)$. Moreover, since $\bigvee\left\{T^{n} x\right\}_{n \geq 0}$ is a nonzero invariant subspace for $T$ whenever $x \neq 0$, it follows that $\bigvee\left\{T^{n} x\right\}_{n \geq 0}=\mathscr{H}$ for each $x \neq 0$ (every nonzero vector in $\mathscr{H}$ is a cyclic vector for $T$ ). Thus the Berger-Shaw theorem (see, for instance, [2, page 152]) ensures that $D$ is a trace-class operator so that $D$ is compact (i.e., $T$ is essentially normal). But for compact operators strong stability coincides with uniform stability, and uniform stability always means spectral radius less than one. Hence the nonnegative $D$ is a strict contraction because it is clearly normaloid (i.e., $\|D\|=$ $r(D)<1)$.

REMARK 3.7. According to the Berger-Shaw theorem, a hyponormal contraction without a nontrivial invariant subspace has a trace-class self-commutator $D$ with tracenorm $\|D\|_{1} \leq 1$. If $D \neq O$ is not a rank-one operator, then $\|D\|<\|D\|_{1} \leq 1$. The above argument ensures the inequality $\|D\|<1$ whenever a hyponormal contraction has no nontrivial invariant subspace, including the case of a hyponormal contraction with a rank-one self-commutator.

An operator is seminormal if it is hyponormal or cohyponormal. Recall that $T^{*}$ has a nontrivial invariant subspace if and only if $T$ has, $T^{*}$ is a proper contraction if and only if $T$ is (Proposition 2.1), and $\left[T, T^{*}\right]=-\left[T^{*}, T\right]$. Thus, the above theorem also holds for cohyponormal contractions. If a seminormal contraction has no nontrivial invariant subspace, then it is a proper contraction and its self-commutator is a strict contraction. This prompts the question: can we drop "hyponormal" from the theorem statement? In particular, is it true that every nonproper contraction has a nontrivial invariant subspace? Theorems 3.4 and 3.6 yield the following result.

COROLLARY 3.8. If a hyponormal contraction $T$ has no nontrivial invariant subspace, then it is either a proper contraction of class $\mathscr{C}_{00}$ or a nonstrict proper contraction of class $\mathscr{C}_{10}$ for which $A$ is a completely nonprojective nonstrict proper contraction. Moreover, its self-commutator $\left[T^{*}, T\right]$ is a strict contraction.

ACKNOWLEDGEMENT. This work was supported in part by CNPq-Brazilian National Research Council.

\section{REFERENCES}

[1] K. N. Boyadzhiev and N. Levan, Strong stability of Hilbert space contraction semigroups, Studia Sci. Math. Hungar. 30 (1995), no. 3-4, 165-182. MR 96g:47034. Zbl 843.47026.

[2] J. B. Conway, The Theory of Subnormal Operators, Mathematical Surveys and Monographs, vol. 36, American Mathematical Society, Rhode Island, 1991. MR 92h:47026. Zbl 743.47012. 
[3] R. G. Douglas, Canonical models, Topics in Operator Theory, no. 13, American Mathematical Society, Rhode Island, 1974, pp. 161-218. MR 50\#8121. Zbl 336.47008.

[4] B. P. Duggal, On unitary parts of contractions, Indian J. Pure Appl. Math. 25 (1994), no. 12, 1243-1247. MR 95m:47012. Zbl 821.47009.

[5] E. Durszt, Contractions as restricted shifts, Acta Sci. Math. (Szeged) 48 (1985), no. 1-4, 129-134. MR 87a:47015. Zbl 588.47013.

[6] P. A. Fillmore, Notes on Operator Theory, Van Nostrand Reinhold Mathematical Studies, no. 30, Van Nostrand Reinhold, New York, 1970. MR 41\#2414. Zbl 216.41601.

[7] P. R. Halmos, A Hilbert Space Problem Book, 2nd ed., Graduate Texts in Mathematics, vol. 19, Springer-Verlag, New York, 1982. MR 84e:47001. Zbl 496.47001.

[8] C. S. Kubrusly, An Introduction to Models and Decompositions in Operator Theory, Birkhäuser Boston, Massachusetts, 1997. MR 98g:47004. Zbl 918.47013.

[9] C. S. Kubrusly and P. C. M. Vieira, Strong stability for cohyponormal operators, J. Operator Theory 31 (1994), no. 1, 123-127. MR 95m:47038. Zbl 832.47018.

[10] C. S. Kubrusly, P. C. M. Vieira, and D. O. Pinto, A decomposition for a class of contractions, Adv. Math. Sci. Appl. 6 (1996), no. 2, 523-530. MR 97h:47017. Zbl 859.47006.

[11] N. Levan, Canonical decompositions of completely nonunitary contractions, J. Math. Anal. Appl. 101 (1984), no. 2, 514-526. MR 86c:47020. Zbl 583.47023.

[12] K. Okubo, The unitary part of paranormal operators, Hokkaido Math. J. 6 (1977), no. 2, 273-275. MR 56\#16427. Zbl 368.47017.

[13] V. Pták and P. Vrbová, An abstract model for compressions, Časopis Pěst. Mat. 113 (1988), no. 3, 252-266. MR 89j:47005. Zbl 669.47005.

[14] C. R. Putnam, Hyponormal contractions and strong power convergence, Pacific J. Math. 57 (1975), no. 2, 531-538. MR 52\#1393. Zbl 321.47018.

[15] M. Shih, P. Tam, and K. K. Tan, Renorms and topological linear contractions on Hilbert spaces, Sci. China Ser. A 42 (1999), no. 3, 246-254. MR 2000f:47037. Zbl 948.46005.

[16] B. Sz.-Nagy and C. Foiaş, Harmonic Analysis of Operators on Hilbert Space, North-Holland Publishing, Amsterdam, 1970. MR 43\#947. Zbl 201.45003.

C. S. Kubrusly: CATHOlic University of Rio De JANeiro, 22453-900 Rio DE JANEIRO, RJ, BRAZIL

E-mail address: car1os@e1e.puc-rio.br

N. LEVAN: UNIVERSiTy OF CALIFORNiA AT Los ANGELES, Los ANGELES, CA 90024-1594, USA

E-mail address: 1evan@ee.ucla.edu 


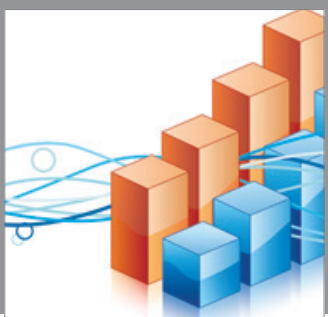

Advances in

Operations Research

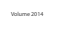

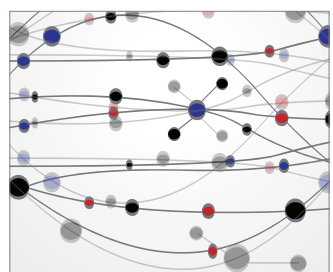

\section{The Scientific} World Journal
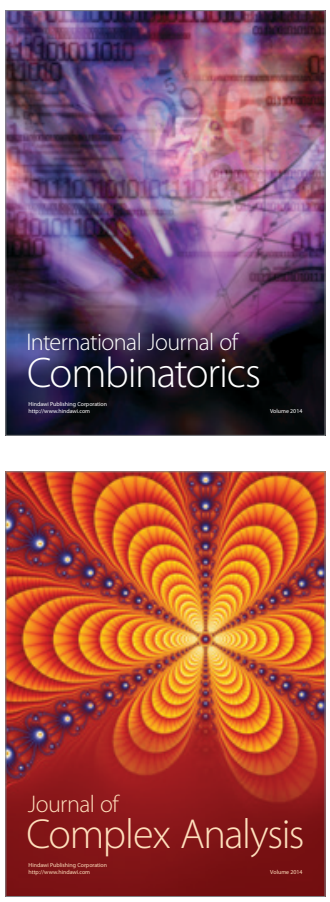

International Journal of

Mathematics and

Mathematical

Sciences
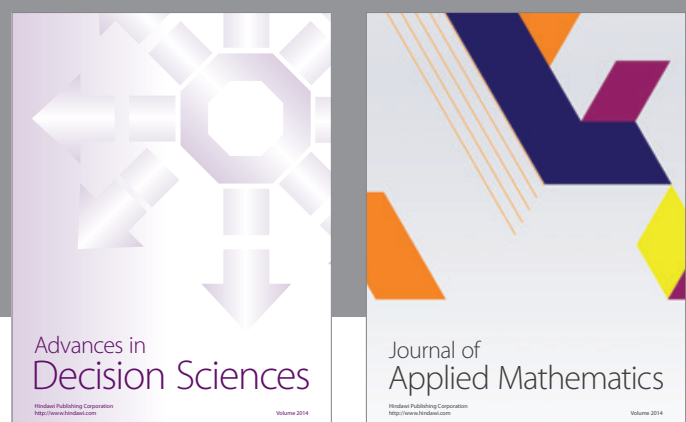

Journal of

Applied Mathematics
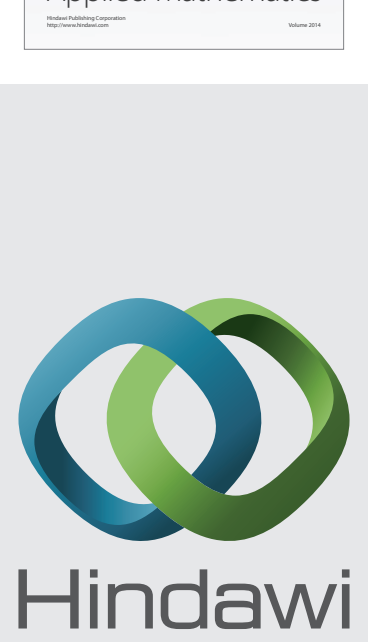

Submit your manuscripts at http://www.hindawi.com
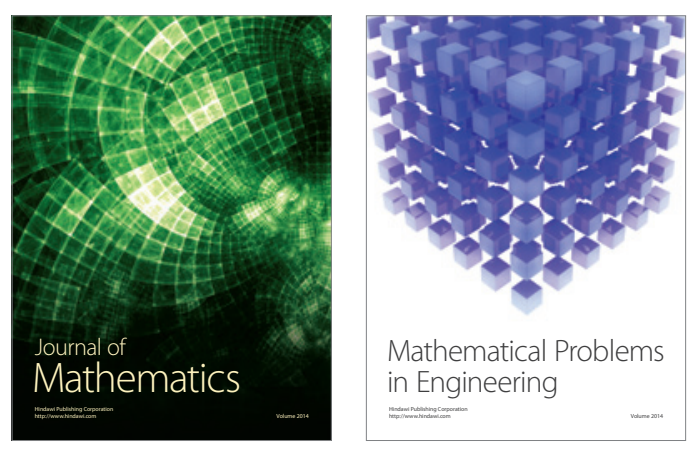

Mathematical Problems in Engineering
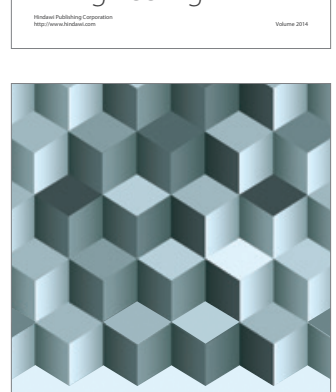

Journal of

Function Spaces
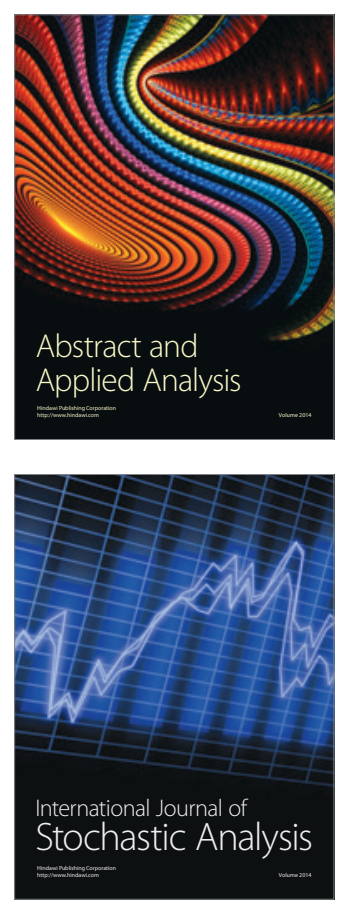

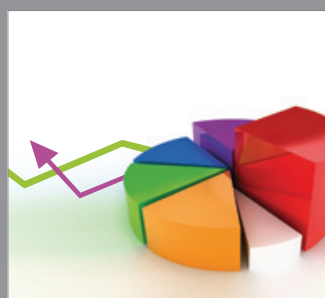

ournal of

Probability and Statistics

Promensencen
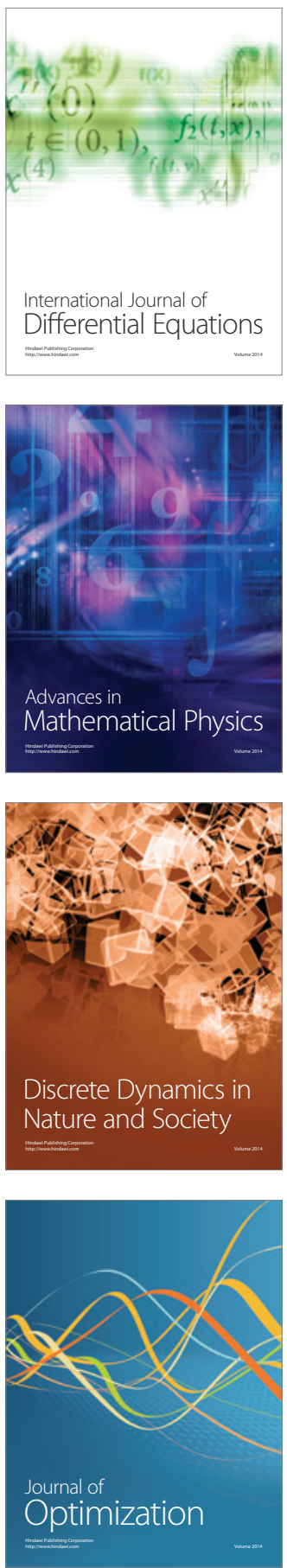